\title{
El miedo a madurar en mujeres con obesidad mórbida postgastrectomía: predictores clínicos
}

Introducción: Analizar la conducta alimentaria en pacientes que son sometidos a cirugía bariátrica. Material y Método: Se administró el Eating Disorder Inventory (EDI) a 85 mujeres con obesidad mórbida intervenidas de cirugía gástrica (gastroplastia vertical bandeada) para perder peso. Resultados: La edad de las pacientes antes de la cirugía y la edad de inicio de la obesidad se relaciona con el Miedo a Madurar del EDI, de forma que cuanto mayor es el paciente y más tarde aparece la obesidad, el miedo a crecer es más intenso. Discusión: Estos hallazgos irían en la dirección de dependencia, inmadurez y miedo a crecer descritos en la población con obesidad mórbida.

Palabras clave: Conducta alimentaria. Obesidad mórbida. Cirugía bariátrica. Predictores clínicos. Rasgos de personalidad.
Introduction: To analyze the eating behaviour in morbidly obese patients undergoing bariatric surgery. Methods: The Eating Disorder Inventory (EDI) was administered to 85 morbidly obese women undergoing gastric surgery (vertical banded gastroplasty) for lossing weight. Results: The age at surgery and the onset obesity age were correlated with maturity fears subescale . Discussion: The findings agree with studies that describe dependence, inmaturity and maturity fears in morbidly obese patients.

Key words: Eating behaviour. Morbid obesity. Bariatric surgery. Clinical predictors. Personality traits.

\section{Introducción.-}

En el desarrollo de la obesidad mórbida se describen la existencia de factores biológicos $(1,2,3)$ y/o factores psicológicos que favorecen la enfermedad $(4,5)$. Aunque ambos factores no son mutuamente excluyentes, parece que los factores genéticos jugarían un papel más importante en la obesidad de inicio en la infancia en tanto que los factores psicológicos influirían más en la obesidad de inicio en la adolescencia/adulto joven. En el primer grupo, cuando la obesidad aparece en la infancia, se ha descrito una mayor refractariedad al tratamiento y a la pérdida de peso, así como una mayor tasa de patología psiquiátrica. Cuando la obesidad se presenta en la adolescencia/adulto joven, se relaciona más con situaciones estresantes a lo largo de la vida, de forma que el enfermo comería en un intento de calmar su malestar psicológico (6).

El objetivo de este estudio es analizar la relación que existe entre la edad en el momento de la intervención quirúrgica y la edad de inicio de la obesidad con la conducta alimentaria de estos enfermos, tras 18 meses de la intervención quirúrgica. 


\section{Material y Método.}

Se administró el Eating Disorder Inventory (EDI) (7) a 85 mujeres con obesidad mórbida que habían recibido intervención quirúrgica (gastroplastia vertical bandeada) para perder peso, 18 meses después de haber sido intervenidas. Dicha entrevista consta de 64 items y mide la conducta alimentaria, peso y silueta corporal, además de factores psicológicos relacionados con los trastornos alimentarios. Consta de 8 escalas: Tendencia a la delgadez, Bulimia, Insatisfacción corporal, Ineficacia, Perfeccionismo, Desconfianza interpersonal, Conciencia Interoceptiva y Miedo a madurar.

Se recoge de cada paciente la edad actual en el momento de la intervención quirúrgica y la edad de inicio de la obesidad. Hemos marcado el punto de corte de la edad de inicio de la obesidad entre 11 y 13 años, para marcar el inicio de la obesidad en la infancia (antes de los 11 años) o en la adolescencia/adulto joven (después de los 13 años). Esta separación ha sido para valorar las diferencias encontradas en el estado mental de los pacientes intervenidos según el inicio de la obesidad.

Apuntamos el Indice de Masa Corporal antes de ser intervenidos y el porcentaje de pérdida de peso tras la cirugía para ver la respuesta al tratamiento quirúrgico:

- Indice de Masa Corporal (IMC): Peso (Kgs) / Altura $\left(\mathrm{m}^{2}\right)$

- Porcentaje de pérdida de peso: (Peso antes de la cirugía- Peso a los 18 meses tras la cirugía) x 100 / Peso antes de la cirugía

Hemos analizado que las variables estudiadas siguen una distribución Normal, tras la realización de los test de Normalidad de Kolmogorov-Smirnov y Shapiro-Wilk. Para el análisis estadístico de los datos se realiza una prueba de $t$ de Student para muestras independientes con la que se analiza la relación que existe entre la edad de inicio de la obesidad y la conducta alimentaria (EDI). Mediante un análisis de regresión lineal (stepwise) se valora la relación entre la edad actual del enfermo y la conducta alimentaria (EDI).

\section{Resultados.}

En la Tabla adjunta, podemos ver las características ponderares y la conducta alimentaria en el conjunto de la muestra y según el inicio de la obesidad.

La edad actual (antes de la cirugía) del paciente se relaciona con el Miedo a madurar (conducta alimentaria valorada a los 18 meses) $(r=0.295, p<0.003)$, de forma que cuanto mayor es el enfermo mayor es el miedo.

\section{Discusión.}

Los pacientes que presentan el inicio de la obesidad en la adolescencia/adulto joven son enfermos susceptibles de una conducta alimentaria alterada en determinadas 
circunstancias como pueden ser estados de disforia y estrés, así como con el consumo de sustancias tóxicas (8). A esta edad la alteración en la imagen corporal, la baja autoestima, la falta de confianza en las situaciones sociales y el estigma de ser obeso marcan la forma de interaccionar con los demás, estando más propensos a ser hostiles en cuanto a su aceptación social (6).

En nuestro estudio nos encontramos con una población de mujeres con obesidad mórbida que han sido intervenidas quirúrgicamente para perder peso. Como grupo, estas enfermas presentan una adecuada pérdida de peso, 18 meses tras la cirugía (> 30\%) lo que indicaría que el tratamiento quirúrgico sigue siendo una adecuada pauta terapéutica en estos enfermos. Asimismo, vemos que presentan altas puntuaciones en insatisfacción corporal y deseo de estar más delgadas, lo que estaría en la dirección de otros estudios que has descrito disfunciones alimentarias en estos enfermos $(8,9)$ que provocan un aumento en la demanda de tratamiento quirúrgico. A mayor edad de los pacientes (antes de la cirugía) el miedo a madurar (conducta alimentaria la cirugía) es mayor y que cuando el inicio de la obesidad es después de los 13 años también existe un mayor temor a crecer. Este miedo crearía un proceso de conflictividad en el adolescente/adulto joven que alteraría el desarrollo de la correcta autonomía que se produce con el crecimiento. Estos enfermos lucharían con los cambios físicos y psíquicos que se producen en esta etapa del desarrollo, apareciendo estructuras de personalidad dependientes e inmaduras, como se han descrito en otros estudios (8-11). Con este procesar, el enfermo presentaría un mayor malestar psicológico buscando con más frecuencia el tratamiento (12). Las enfermas que han iniciado la obesidad en la adolescencia/adulto joven presentan menos peso y mayor edad antes de la cirugía, con mayor sensación de ineficacia y miedo a madurar tras la intervención quirúrgica. Estos datos están en corcordancia con los estudios que han encontrado que a mayor peso y edad más joven antes de la intervención, el resultado postquirúrgico es mejor (13) por lo que nuestras enfermas apuntarían a un peor pronóstico, si además añadimos, que presentan elementos de ineficacia y un intenso miedo a madurar un vez intervenidas. Este grupo solicitó el tratamiento con menos peso antes de ser intervenidas lo que aporta que tienen mayor malestar psicológico, el cual aumentaría la petición del tratamiento quirúrgico (12) evolucionando peor tras la operación.

Dado los hallazgos de este estudio, parece que la edad (antes de la cirugía y de inicio de la obesidad) serían dos predictores clínicos en la conducta alimentaria de nuestros pacientes con obesidad mórbida una vez intervenidos. El análisis de los factores psicológicos asociados a la conducta alimentaria de estas pacientes, como puede ser el miedo a madurar, es de vital importancia para una adecuada pérdida de peso tras la cirugía. 


\begin{tabular}{|c|c|c|c|c|c|c|c|c|}
\hline \multirow[t]{2}{*}{ Items } & \multicolumn{2}{|c|}{ Total $(n=85$} & \multicolumn{5}{|c|}{$\begin{array}{c}\text { Inicio }<10 \text { años Inicio }>13 \text { años } \\
\quad(n=25) \quad(n=60)\end{array}$} & \multirow[b]{2}{*}{$\mathrm{P}$} \\
\hline & Media & $\mathrm{DE}$ & Media & $\mathrm{DE}$ & Media & $\mathrm{DE}$ & $\mathrm{t}$ & \\
\hline IMC antes de cirugía & 49.09 & 8.04 & 51.53 & 9.23 & 47.77 & 6.99 & 2.227 & 0.05 \\
\hline$\%$ Pérdida de peso & 31.32 & 10.39 & 32.56 & 12.08 & 30.67 & 9.64 & & n.s \\
\hline Edad antes de cirugía & 40.50 & 11.15 & 35.73 & 10.91 & 42.58 & 10.76 & -2.900 & 0.001 \\
\hline Tendencia a la delgadez & 8.33 & 5.36 & 7.36 & 5.42 & 8.75 & 5.36 & & n.s \\
\hline Bulimia & 1.21 & 2.40 & 1.40 & 3.11 & 1.14 & 2.05 & & n.s \\
\hline Insatisfación corporal & 14.59 & 8.87 & 15.36 & 9.38 & 14.31 & 8.73 & & n.s \\
\hline Ineficacia & 3.53 & 4.85 & 2.10 & 3.83 & 4.20 & 5.14 & -2.249 & 0.05 \\
\hline Perfeccionismo & 5.24 & 3.42 & 4.86 & 3.15 & 5.40 & 3.56 & & n.s \\
\hline Desconfianza interpersonal & 3.09 & 3.01 & 2.83 & 3.66 & 3.23 & 2.71 & & n.s \\
\hline Conciencia interoceptiva & 5.44 & 4.92 & 4.33 & 4.63 & 5.97 & 5.01 & & n.s \\
\hline Miedo a madurar & 5.89 & 4.18 & 4.63 & 3.56 & 6.46 & 4.36 & -2.021 & 0.01 \\
\hline$\overline{\text { Edi total }}$ & 5.91 & 2.88 & 5.36 & 2.83 & 6.18 & 2.90 & & n.s \\
\hline
\end{tabular}

TABLA: Características generales de la muestra

$\mathrm{t}$ (Prueba t de Student)

p (probabilidad estadística) 


\section{BIBLIOGRAFÍA.}

1. Daniels JS. "The pathogenesis of obesity". Psychiatr Clin North Am 1984; 7:335-347.

2. Weiss SR. "Obesity-pathogenesis, consequences and approaches to treatment". Psychiatr Clin North Am 1984; 7: 307-319.

3. Price RA. "Genetics of human obesity". Ann Behav Med 1987; 9: 9-14.

4. Kaplan HI, Kaplan HS. "The psychosomatic concept of obesity". J Nerv Ment Dis 1957; 125:181-201.

5. Bruch H. Eating disorders. London: Loutledge \& Keegan Paul, 1974.

6. Mills JK. "A note on interpersonal sensitivity and psychotic symptomatology in obese adult outpatients with a history of chilhood obesity". The Journal of Psychology 1995; 129: 345-348.

7. Garner DM, Olmsted MP, Polivi J. "Development and validation of a multidimensional Eating Disorder Inventory for anorexia nervosa and bulimia". Int J Eat Disord 1983; 2: 15-33.

8. Larsen F and Torgersen S. "Personality changes after gastric banding surgery for morbid obesity. A prospective study". Journal of Psychosomatic Research 1989; 3: 323-334.

9. Karlsson J, Sjöström L, and Sullivan M. "Swedish obese subjects (SOS)-an intervention study of obesity. Two-year follow-up of health related quality of life (HRQL) ans eating behavior after gastric surgery for severe obesity". Int J Obes 1998; 22: 113-126.

10. Abraham HS, Meixel SA, Webb WW, scott HW. "Psychological adaption to jejunoileal for morbid obesity". J Nerv Ment Dis 1976; 162: 151-157.

11. Castelnuovo-Tedesco P, Schiebel P. "Studies of superobesity: I. Psychological characteristics of superobese parients". Int J Psychiatr Med 1975; 6: 465-480.

12. Cummings NA, Follette WT. "Psychiatric services and medical utilization in a prepaid plan setting: Part II". Med Care 1968; 6: 31-41.

13. Barrash J, Rodriguez EM, Scott DH, Mason EE, Sines JO. "The utility of MMPI subtypes for the prediction of weight loss after bariatric surgery”. Int J Obes 1987; 11: 115-128.

*Departamento de Psiquiatría. Facultad de Medicina. Universidad de Extremadura. Badajoz.

Correspondencia:

Juan Antonio Guisado Macías,

$\mathrm{C} /$ Rafael Lucenqui $\mathrm{n}^{\circ} 10,6^{\circ} \mathrm{F}$,

06004, Badajoz,

e-mail: jguisadom@medynet.com

Fecha de recepción: 30-01-02 